ENSAYO 



\section{CALIDAD EN SALUD Y SEGURIDAD DEL PACIENTE, ¿ENTENDEMOS LO QUE SIGNIFICA?}

ANA MILENA MINA OSORIO

Recibido: 22 de febrero de 2019 Aceptado: 1 de noviembre de 2019 



\title{
CALIDAD EN SALUD Y SEGURIDAD DEL PACIENTE, ¿ENTENDEMOS LO QUE SIGNIFICA?
}

\author{
Ana Milena Mina Osorio
}

Empecemos hablando del concepto de calidad en salud, el cual se ha implementado paulatinamente en los países latinoamericanos, por la falta de recursos, y de conocimientos de base.

En salud, la gestión de la calidad implica una serie de procesos interrelacionados: desde la función directiva de planificación estratégica y toma de decisiones oportunas, hasta la función operativa encargada de realizar los procesos organizativos o logísticos. Todo se lleva a cabo mediante un trabajo en equipo, de corte multidisciplinario, bajo una misma consigna: la satisfacción del cliente.

Los pacientes son el eje hacia quienes deberían dirigirse todos nuestros esfuerzos, pues ellos son la razón de ser, el núcleo de todos los que trabajamos en salud. Por tanto, la humanización de la asistencia en salud, debe ser el enfoque principal de la gestión de la calidad.

Promover la calidad significa hacer las cosas bien. Dentro de esta noción se integran, principalmente, la seguri- dad del paciente como una generación de cultura y la organización, entendida como la toma de conciencia de la necesidad de seguir procedimientos que prevengan el daño en el paciente que, en ocasiones, podría ser irreversible.

La Organización Mundial de la Salud (OMS) define: "la calidad de la asistencia sanitaria es asegurar que cada paciente reciba el conjunto de servicios diagnósticos y terapéuticos más adecuados para conseguir una atención sanitaria óptima, teniendo en cuenta todos los factores y los conocimientos del paciente y del servicio médico, y lograr el mejor resultado con el mínimo riesgo de efectos iatrogénicos y la máxima satisfacción del paciente con el proceso"1.

Debido a la necesidad de promover una adecuada atención en salud, se creó un programa de concientización de la prevención del daño en la atención sanitaria en todo el personal de salud. De esta forma, se generaron una serie de actividades encaminadas a minimizar el riesgo de que ocurra un evento que cau- 
se un efecto no deseable en el paciente.

Sin embargo, este tema no es nuevo. Ya en 1999, el Instituto de Medicina (IOM) en Estados Unidos publicó el informe: "To Err is Human: building a Safer Health System"2. Su contenido provocó un gran impacto en la sociedad -y en la propia comunidad médica - al señalar que la mortalidad de pacientes hospitalizados por errores médicos oscilaba entre 44.000 y 98.000 por año, por arriba de accidentes automovilísticos, cáncer de mama o SIDA.

Este informe fue precedido por el estudio Harvard realizado en New York, en el año 1984, Ilamado Harvard Medical Practice Study, el cual estimó una incidencia de 3,7 \% de eventos adversos, de los cuales el $14 \%$ condujeron, lamentablemente, a la muerte ${ }^{3}$.

La OMS publica en su reporte: 10 datos sobre seguridad del paciente:

- Uno de cada 10 pacientes puede sufrir algún tipo de daño en el hospital.

- Las infecciones hospitalarias afectan a 14 de cada 100 pacientes ingresados.

- La mayoría de las personas no tiene acceso a dispositivos médicos apropiados.

- $\quad$ Entre el $20 \%$ - $40 \%$ de todo el gasto en salud se despilfarra a causa de una atención de calidad deficiente.

- La probabilidad de que un pasajero sufra algún daño en un avión es de 1 en 1000 000. En cambio, la probabilidad de que un paciente sufra algún daño ocasionado por la atención médica es de 1 en $300^{4}$.

En la Asamblea Mundial de la Salud de 2002, los Estados Miembros de la OMS adoptaron una resolución sobre la seguridad del paciente. Desde la puesta en marcha en 2004 del Programa de Seguridad del Paciente de la OMS, más de 140 países han tratado de solucionar los problemas que plantea una atención sin garantía de seguridad. Actualmente, es notable que la seguridad del paciente y la calidad de la atención que se le presta son dimensiones fundamentales de la cobertura sanitaria universal.

- Las inyecciones sin garantía de seguridad se han reducido en un $88 \%$ entre 2000 y 2010.

- El trabajo en equipo es imprescindible para que las intervenciones quirúrgicas sean seguras.

- La atención médica debe estar centrada en los pacientes. Su retroalimentación es fundamental para garantizar la seguridad en la atención.

- Las alianzas de hospitales pueden desempeñar una función crucial ${ }^{4}$.

En América Latina, se realizó el estudio IBEAS: Prevalencia de Efectos 
Adversos, en 58 hospitales de cinco países: México, Perú, Argentina, Costa Rica y Colombia, con una muestra de 11.555 pacientes.

La prevalencia de eventos adversos encontrada en el estudio, fue de
10,5\%. Los más frecuentes fueron aquellos relacionados a infección nosocomial. Lo destacable del estudio es que un $60 \%$ de los eventos adversos presentados pudo haberse evitado 5 .

\section{REALIDAD NACIONAL}

En el Ecuador, se implementa a través del Ministerio de Salud Pública (MSP), el Manual de Seguridad del Paciente - Usuario, en el año 2016, Acuerdo Ministerial 115, indicando como disposición transitoria en el numeral 1, la aplicación del mismo en todo el Sistema Nacional de Salud'.
Hasta el momento, no se cuenta con un registro oficial publicado que nos provea de datos en relación a la prevalencia de eventos adversos en el país, ni del impacto de la implementación de dicho manual en el Sistema Nacional de Salud.

\section{DEFINICIÓN}

Con todo lo mencionado anteriormente, podemos definir qué es la Seguridad del paciente.

Podríamos, entonces, describir a la seguridad del paciente como todos los actos dentro de la atención sanitaria que garanticen o aseguren buenas prácticas clínicas, que prevengan o eviten un daño en el paciente y que no sean originados por su enfermedad de base.

Según la OMS, la definición de Seguridad del Paciente es el hecho de estar a salvo de lesiones accidentales.
Velar por la seguridad del paciente supone instaurar sistemas y procesos operacionales que reduzcan al mínimo la probabilidad de errores y maximicen la probabilidad de interceptarlos cuando ocurran7; o, simplemente, el hecho de que un paciente esté a salvo de daños innecesarios o potenciales asociados a la atención sanitaria.

Resta preguntarnos ¿a qué se llama evento adverso, cuasi incidente y evento centinela?:

Evento adverso: es una acción no 
intencional que produce daño al paciente dentro de la atención sanitaria.

Cuasi Incidente: es una circunstancia que no produjo daño al paciente, sea por casualidad o por medidas de prevención adoptadas en el momento.

Evento centinela: es la acción que ocasiona la muerte o daño permanente del paciente debido a la atención sanitaria.

\section{EVALUACIÓN}

Se debe considerar que un evento adverso es producido por múltiples causas, pues no es consecuencia de la actuación de una sola persona, sino que puede ser producto de la suma de otros factores, que se denominan causas próximas.
Una forma de análisis es, por ejemplo, el análisis causa raíz o espina de pescado de Ishikawa, que busca, través de una investigación sistemática basada en una serie de preguntas, la determinación de la causa que originó el evento adverso para prevenir o disminuir su aparición ${ }^{6}$.

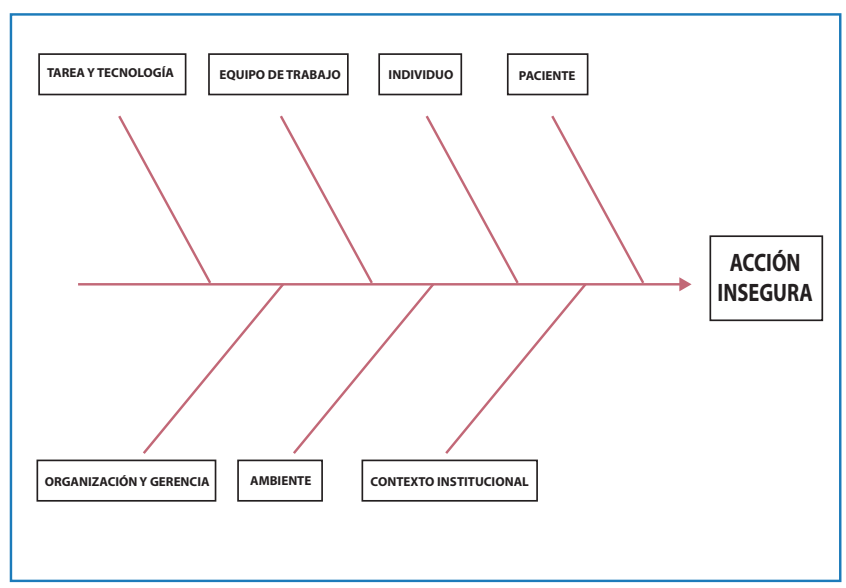

Tomado de la Guía de buenas prácticas en seguridad del paciente. Ministerio de la Protección Social. Colombia. 
Debido a estas particularidades, se empezó a utilizar el Protocolo de Londres, llamado también Modelo de Multicausalidad Organizativo de Accidentes, que fue aplicado inicialmente en la aviación e industria petrolera, para la prevención de accidentes, basado en el modelo del error de Reason.

Este método es similar al análisis de causa raíz. La diferencia radica en que este último se usa más en análisis de evento centinela, mientras que el protocolo de Londres puede ser usado en cualquier evento adverso, incluso en incidentes.

\section{Consta de siete fases:}

1a Fase:

Identificar y decidir qué investigar 2a Fase:

Selección del equipo investigador $3^{\text {a }}$ Fase:

Obtener y organizar la información 4a Fase:

Establecer la cronología del incidente 5a Fase:

Identificar las acciones inseguras

6a Fase:

Averiguar los factores contribuyentes 7a Fase:

Elaborar un plan de acción con recomendaciones $^{7}$

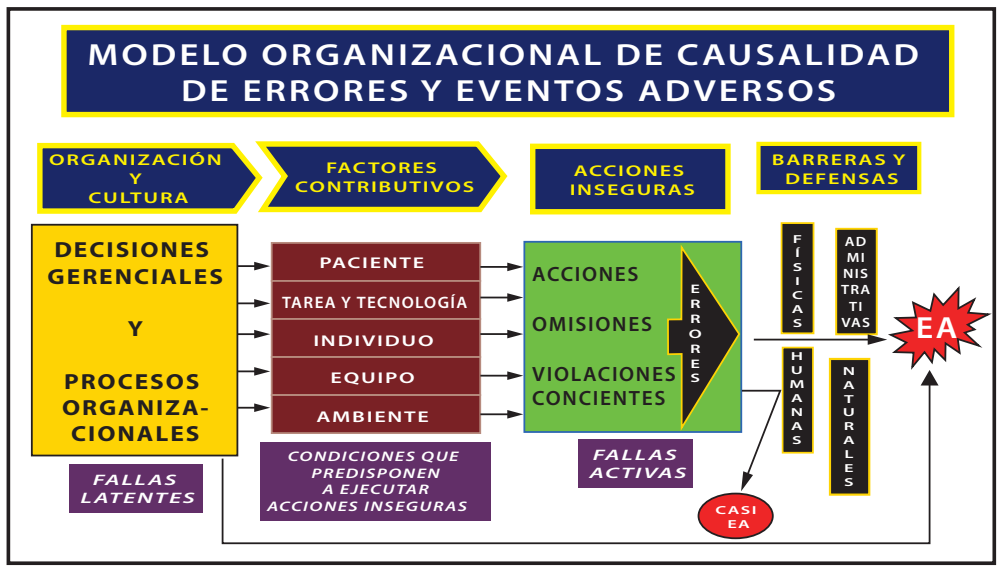

Tomado the London protocol. Autores: Sally Taylor-Adams y Charles Vincent (Clinical Safety Research Unit, Imperial College. London, UK). 
Una vez realizados todos los pasos del protocolo, se pueden verificar las acciones inseguras que se cometieron en cada uno de ellos, llegando a la conclusión de que no siempre es un solo factor el que ocasiona el evento adverso, sino que son varios los factores que con- tribuyen a su aparición. No obstante, lo más importante es destacar y reconocer que pueden ser controlados, mejorando los procesos instaurados en las organizaciones, con planes de acción bien establecidos que permitan evitarlos.

\section{CONCLUSIONES}

La calidad de la atención es fundamental para asegurar el correcto proceso diagnóstico, terapéutico y de prevención en salud. Por tanto, su gestión en las organizaciones es imprescindible.

La cobertura universal de salud implica que todas las personas y comunidades puedan usar los servicios de promoción de la salud, prevención, tratamiento, rehabilitación y cuidados paliativos que necesitan, de calidad suficiente para ser eficaces, y a la vez asegurar que su uso no exponga a dificultades financieras al usuario. Muchos países están realizando esfuerzos para mejorar la calidad de la atención e institucionalizar una cultura de la calidad en todo su sistema de salud. Esos esfuerzos pueden fortalecerse mediante la formulación de políticas y estrategias nacionales de calidad, que deberían replantearse en nuestro país. ${ }^{8}$

La seguridad del paciente debe ser concebida desde la cultura de se- guridad a implementarse en las organizaciones como una cultura no punitiva, en la cual el personal de salud se sienta respaldado en el momento de hacer un reporte de un incidente o evento adverso para, de esta forma, instaurar todas las medidas preventivas necesarias.

La cultura se expresa de muchas maneras, en especial a través de los valores, creencias, actitudes, conductas, lenguajes, costumbres, metas, políticas y operaciones de los diferentes grupos de trabajo. Además, crea un sentido de identidad y establece vínculos vitales entre los miembros de una organización y su misión. Referido a la salud es el producto de interacciones dinámicas que, a nivel de la atención al paciente, deben integrarse bajo principios éticos y científicos y permear todos los niveles de la organización hospitalaria, para elevar el nivel de la misión institucional. ${ }^{9}$ 
Se deben realizar propuestas de intervención en las organizaciones de salud, para mejorar el clima de seguridad, entre ellas ${ }^{10}$ :

- Fomentar la notificación interna y discusión de los errores que puedan ocurrir: La clara actitud positiva para aprender de ellos, es una fortaleza que debe ser aprovechada.

- Tratar los temas de seguridad del paciente como un problema de equipo, no individual: La buena percepción de la importancia de la interrelación interna en las diversas Unidades o Servicios debe ser asimismo aprovechada.

- Prestar atención prioritaria a la racionalidad de la dotación y organización del personal y los ritmos de trabajo a que se puedan ver sometidos: En este ámbito, es donde se percibe la existencia de debilidades u oportunidades de mejora.

- Fomentar la responsabilidad compartida y la coordinación entre Unidades y Servicios: Particularmente en hospitales de tamaño grande y mediano se percibe la necesidad de mejorar la coordinación entre unidades y servicios y debería por tanto desarrollarse una estrategia tendente a hacer extensiva la sensación de trabajo en equipo entre todas las unidades.
- Mostrar una actitud más claramente proactiva hacia la seguridad del paciente por parte de la gerencia.

No existe calidad sin evaluación. Por tanto, la evaluación de todos los procesos instaurados debe ser permanente, para tomar decisiones que permitan corregir posibles fallas que terminen en daño o lesión ocasionados por un mal proceso de atención sanitaria.

La Acreditación, como forma de evaluación en salud, implica e incentiva a los profesionales en la mejora continua de la calidad, fomenta la interdisciplinariedad, supone un prestigio para los que trabajan en centros asistenciales acreditados, otorgando ventajas a la organización, como son: Enfocar al paciente como eje central, garantizar sus derechos y fomentar su seguridad y proporcionar además información objetiva sobre los distintos centros asistenciales, para la toma de decisiones locales, regionales o nacionales ${ }^{11}$.

Es fundamental entonces, que todas las organizaciones de salud implementen procesos de calidad en la atención sanitaria, como parte de su rutina laboral, para disminuir y evitar la aparición de eventos adversos, que ocasionen daño en el paciente, que no siempre es producido intencionalmente por parte del personal, sino como consecuencia de la ausencia de procesos clínicos, que prevengan su aparición. 


\section{REFERENCIAS}

1. Organización Mundial de la Salud. 2018. Manual para la elaboración de políticas y estrategias nacionales de calidad. Un enfoque práctico para elaborar políticas y estrategias destinadas a mejorar la calidad de la atención. Recuperado de: http://iris.paho. org/xmlui/handle/123456789/49549

2. Institute of Medicine. Recuperado de: http://www.nationalacademies.org/ hmd/Reports/1999/T

3. Brennan TA, Leape $L L$, Laird NM, et al. 1991. Incidence of adverse events and negligence in hospitalized patients: results of the Harvard Medical Practice Study I. N Engl J Med;324:370-7. Recuperado de: https://www.nejm.org/doi/ full/10.1056/NEJM199102073240605

4. Organización Mundial de la Salud. 2002. 10 datos sobre Seguridad del paciente. Recuperado de :http:// www.who.int/features/factfiles/patient_safety/patient_safety_facts/es/

5. Aranaz, Jesús María, Aibar Remon Carlos, et al. 2010. Estudio Ibeas. Prevalencia de Eventos Adversos en hospitales de Latinoamerica. OMS, Ministerio de Sanidad y Política Social de España. Recuperado de: http://new. paho.org/hq/dmdocuments/2010/ INFORME\%20GLOBAL\%20IBEAS.pdf

6. Guadalupe Olivera Cañada, José Sau- ra Llamas et al. 2003. Revista Clínica Electrónica en Atención Primaria. Núm. 18. Universidad Autónoma de Barcelona. España. Recuperado de: http://calidadasistencial.es/images/ gestion_soc/documentos/232.pdf

7. Sally Taylor-Adams y Charles Vincent. 1999. Protocolo de Londres. (Clinical Safety Research Unit, Imperial College. London, UK). Recuperado de: https://www.minsalud.gov.co/sites/ rid/Lists/BibliotecaDigital/RIDE/DE/ CA/PROTOCOLO_DE_LONDRES_INCIDENTES\%20CLINICOS.pdf

8. Gómez O, Arenas W, González L, Garzón J, Mateus E, Soto A. 2011.Cultura de seguridad del paciente por personal de enfermería en Bogotá, Colombia. Ciencias y enfermería,:1-15. Recuperado de: http://www.scielo.cl/pdf/ cienf/v17n3/art09.pdf

9. Mira J, Cho M, Montserrat D, Rodríguez J. 2013. Elementos clave en la implantación de sistemas de notificación de eventos adversos hospitalarios en América Latina. Rev. Panam Salud Pública.1-7. Recuperado de: http://bit.ly/SS9rCc

10. Álvarez C, 2010. La prevención de las infecciones asociadas a la atención en salud: un reto en el mejoramiento de la seguridad del paciente.1-2. Recuperado de: http://revistainfectio. 
org/site/portals/0/ojs/index.php/infectio/article/view/62

11. Villareal E. 2007. Seguridad de los pacientes. Un compromiso de todos para un cuidado de calidad. Salud Uninorte. pág. 1-8. Recuperado de: http://bit.ly/1q90DEo

12. Martinez A, Hueso C, Galves M, Soto A. 2010. Fortalezas y amenazas en torno a la seguridad del paciente según la opinión de los profesionales de enfermería, pág.1-8. Recuperado de: http://www.scielo.br/pdf/rlae/ v18n3/es_07.pdf

13. Wegner W, Rubin E. 2012. La seguridad del paciente en las circunstancias de cuidado: prevención de eventos adversos en la hospitalización infantil pág.1-8. Recuperado de: http://www.scielo.br/pdf/rlae/ v20n3/es_a02v20n3.pdf

14. Escobar F, Sabóia M, Alux T, Bauer A, Perufo S, De Bortoli S. 2010.Seguridad del paciente en la terapéutica medicamentosa y la influencia de la prescripción médica en los errores de dosis. Revista Latinoamericana Enfermagem. 18(6): pág.1-7. Recuperado de: http://www.scielo.br/pdf/rlae/ v18n6/es_03.pdf

15. Mena P. 2008. Error médico y eventos adversos. Revista Chilena de Pediatría. 19 (3)319-326 Recuperado de: http://bit.ly/1nzN67c

16. Muiño A, Jiménez $A$, Muñoz, Pinilla
LI, Durán M, F, Cabrera F, Rodríguez M. 2007. Seguridad del paciente,: pág.15. Recuperado de: http://repositorio. ucm.edu.co:8080/jspui/bitstream/ handle/10839/761/Maria\%20Consuelo\%20Prado\%20Cuellar.pdf?sequence $=1$ \&isAllowed $=y$

17. Gómez O, Soto A, Arenas A, Garzón J, González A, Mateus E. Una mirada actual de la cultura de seguridad del paciente. Avances en Enfermería. 29 (2) pág.1-12. Recuperado de: http:// bit.ly/1udWFcM

18. Toffoletto M, Ramírez X. 2.013. Mejorando la seguridad de los pacientes: estudio de los incidentes en los cuidados de enfermería. Rev. esc. Enfermería.: vol.47. pág.1098 a 1105. Recuperado de: http://bit.ly/1 kZLmnR

19. López M, Alber M, Jiménez N. 2013. Medida de la mejora continua en la seguridad del paciente oncológico. Farm Hosp.37 (2): pág.143-150. Recuperado de: http://www.ncbi.nlm.nih. gov/pubmed/23789758 (14)

20. Mira J, Lorenzo S, Vitaller J, Ziadi M, Ortiz L, Ignacio E, Aranaz J. 2009. El punto de vista de los pacientes sobre la seguridad clínica de los hospitales. Validación del Cuestionario de Percepción de Seguridad. Rev. méd. Chile. vol.137, pág.1441-1448. Recuperado de: http://bit.ly/1kZG1gq

21. Betancourt J. Cepero R, Gallo C. 2011. Factores que puede afectar la segu- 
ridad del paciente. AMC., vol.15, pág. 936-945. Recuperado de: http://bit. ly/1qkwMYw

22. Ortells A, Paguina M. 2012. Indicadores de calidad y seguridad del paciente en la enfermería de urgencias: un valor seguro. Enfermería Global. 11 (26)pág.184-190. Recuperado de: http://bit.ly/1kZGf7c 22

23. Machado J, Marques A, Martins M. 2013. Evaluación de la calidad de la atención hospitalaria en el Brasil: una revisión sistemática.Cadernos de Saude. 29 (6). pág. 1063-1082. Recuperado de: http://www.scielosp. org/scielo.php?script=sci_arttext\&pi$d=S 0102311 \times 2013001000004$

24. Amaya A, Narváez J, Schmalbach E. 2013. Trabajo en equipo como factor contribuyente en la ocurrencia de errores médicos o eventos adversos. Rev. colomb. De Cirugía. 28 (4)pág. 297-310. Recuperado de http://bit. ly/1s1xEpv

25. Bathke J, Cunico P, Maziero E, Frates F, Mansano L, Almeida D. 2013. Infraestructura y la adhesión a la higiene de manos: desafíos para la seguridad del paciente. Rev. Gaúcha Enferm. 34 (2); pág. 78-85. Recuperado de: http://bit. ly/1 kfBIJ8

26. Cararro T, Galce F, Sebil L, Kepler S, Capelina M, Waterkemper R. 2012. La bioseguridad y la seguridad del paciente a la vista de los estudiantes de enfermería. Rev. Gaúcha Enferm 33 (3) pág. 14-19. Recuperado de: http:// bit.ly/1s1zK8Z

27. Godinho M, Carvalho R, Lima R, Cassiani S, Galon T. 2012. El clima de la seguridad del paciente: la percepción de los profesionales de enfermería.Acta Paulista de Enfermagem. 25 (5). Pág.728-735. Recuperado de: http://bit.ly/1kV90mE

28. Bohomol E, Ramos L. 2007. Los errores de medicación: la importancia de la notificación en la gestión de seguridad del paciente. Rev. bras. enferm. 60 (16). pág. 32-36. Recuperado de: http://bit.ly/1pY4S7g

29. Aranaz Jesus María. 2.010. IBEAS, Estudio de prevalencia de efectos adversos en hospitales de Latinoamérica. Madrid - España. Ministerio de Sanidad. Recuperado de: http://bit. ly/1l2glek

30. Ministerio de la Protección Social. 2014. Guía técnica "Buena práctica para la seguridad del paciente en la atención en salud".Bogotá - Colombia. Recuperado de: https:// www.minsalud.gov.co/sites/rid/ Lists/BibliotecaDigital/RIDE/DE/ CA/Guia-buenas-practicas-seguridad-paciente.pdf

31. Organización Mundial de la Salud; 2007. Centro Colaborador de la OMS sobre Soluciones para la Seguridad del Paciente.. Recuperado de: https:// 
www.who.int/mediacentre/news/releases/2007/pr22/es/

32. Roqueta F;Tomas S, Cánovas M. 2012. Cultura de seguridad del paciente en los servicios de urgencias: resultados de su evaluación en 30 hospitales del Sistema Nacional de Salud español. Revista de Emergencias. 24: 225-233. Recuperado de: http://www.semes. org/revista/vol23_5/4.pdf

33. Álvarez J. Portella Enrique T, Fernández M. 2007. Practicas Seguras Simples. Ministerio de Sanidad de España. Recuperado de: http://bit. ly/107n2ni

34. Fajardo G.; Rodríguez J.; Arboleya H.; Rojano C.; Hernández F.; Santacruz J. 2010. Cultura sobre seguridad del paciente en profesionales de la salud. Revista Cirugía Cirujanos. No. 6., pág. 6. http://bit.ly/107r27u

35. Saturno P, Gama D, Sousa S. Fonseca A. 2008. Análisis de la cultura sobre seguridad del paciente en los hospitales del Sistema Nacional de Salud Español. Ministerio de Sanidad Español.; pág.18-23. Recuperado de: http://www.mscbs.gob.es/organizacion/sns/planCalidadSNS/docs/ Analisis_cultura_SP_ambito_hospitalario.pdf

36. Fajardo, G. Philippe L, Rodriguez J. Fuentes M. 2010. Sistema de registro electrónico de incidentes, basado en la Clasificación Internacional para la
Seguridad del Paciente de la Organizacional Mundial de la Salud. Revista Conamed. 15 (1) pág.29-36. Recuperado de: https://dialnet.uniroja.es

37. Romero O.; García M.; Fernández M.; Ramos J.; Guerrero J.; Mármol R. 2012. La seguridad del paciente como indicador de la calidad asistencial y del trabajo enfermero. El empleo del "listado pre quirúrgico", pág. 6. Recuperado de: http://www.scielo.cl/pdf/ cienf/v17n3/art09.pdf

38. Fernández E, Romero A, Plaza M, García M. 2007.. Seguridad del paciente: protocolo de contención mecánica e inmovilización de pacientes. Hospital Universitario San Cecilio de Granada. Andalucía - España. Recuperado de: sb86eb09335ad47f5.jimcontent.com

39. Bernal, M; Pena, L; Escudero, S. $2011 .$. Plan estratégico de seguridad del paciente. pág.1-98. Recuperado de: http://bit.ly/1xAqqZS

40. Santiago T, Chanovas M, Raqueta F. 2012. La seguridad del paciente en urgencias y emergencias: balance de cuatro años del Programa SEMES-Seguridad Paciente. Recuperado de: http://www.semes.org/revista/ vol24_3/11.pdf

41. Barroeta, J; Sanchez, C. 2008. Guía de práctica clínica de Seguridad del paciente. Empresa Pública de Emergencias Sanitarias. Andalucía - España. pág.1-64. Recuperado de: http:// 
www.epes.es/wp-content/uploads/ Guxa_Prxctica_Seguridad_del_Paciente-2ed.pdf

42. Zenewtin, A, Catarina A, Saturno P. 2013. Cultura de seguridad del paciente y factores asociados en una red de hospitales públicos españoles. Cadernos de Saude Publica. 29 (2). http://www.scielosp.org/pdf/csp/ v29n2/15.pdf

43. Betancourt D, Rodriguez C, Cuervo C. 2008. Lineamientos para la implementación de la política de seguridad del paciente en la república de Colombia. Recuperado de: http://bit. ly/1xAsu4f

44. Luengas S. 2009. Seguridad del paciente: conceptos y análisis de eventos adversos. pág.1-13. Recuperado de: http://www.conamed.gob.mx/ gobmx/boletin/pdf/boletin3/numero_completo.pdf

45. Quadrado E, Tronchin D. 2012. Evaluación del protocolo de identificación de los recién nacidos en un hospital privado. Rev. Latino-Am. De Enfermagem. 20(4) http://www.scielo.br/pdf/rlae/v20n4/es_05.pdf

46. Schatkoski A, Wegner W, Algeri S, Rubim N. 2009. Seguridad y protección para el niño hospitalizado: estudio de revisión. Revista Lationoam Enfermagem. 17(3) Recuperado de: http:// www.scielo.br/pdf/rlae/v17n3/ es_20.pdf
47. Raduenz A, Hoffmann P, Radunz V, Dal Sasso G, Maliska I, Marck P. 2010. Cuidado de enfermería y seguridad del paciente: visualizando la organización, acondicionamiento y distribución de medicamentos con método de investigación fotográfica. Rev. Latino. 18(6). Pág. 8-10. Recuperado de: http://www.scielo.br/pdf/rlae/ v18n6/es_02.pdf

48. Jardali, F. Sheikh F. 2014. Cultura de seguridad del paciente en un gran hospital universitario en Riyadh: evaluación inicial, el análisis y las oportunidades de mejora comparativa. Recuperado de: http://www.biomedcentral.com/1472-6963/14/122

49. Oliveira, M; Goncalves M; Sorgini M. 2013. Cirugía segura en pediatría: elaboración y validación de checklist de intervenciones preoperatorias. pág. 2-8. Recuperado de: www. revistas.usp.br/rlae/article/download/76025/79663

50. Okuyama A, Wagner C, Bijnen B. 2014. En declaraciones para la seguridad del paciente por los profesionales de la salud en los hospitales: una revisión de la literatura. Recuperado de: http://www.ncbi.nlm.nih.gov/pubmed/24507747

51. El-Jardali F, Jaafar M, Dimassi H, Jamal D, Hamdan R. 2010. El estado actual de la cultura de seguridad del paciente en los hospitales Libaneses: 
un estudio al inicio del estudio. International Journal for Quality in Health Care; Volume 22, pág. 386- 395. Recuperado de: http://www.ncbi.nlm. nih.gov/pubmed/20699233

52. Mohsin S, Garfield S, Dean B. 2014. Participación de los pacientes en la seguridad de medicamentos en el hospital: un estudio exploratorio. Rev Int JClin Pharm. Recuperado de: http://www.ncbi.nlm.nih.gov/pubmed/24777838
53. Sunol R, Wagner $C$, Arah $O$, Shaw $C$, Kristensen S, Thompson C, Dersarkissian $M$, Bartels P, Pfaff $H$, Secanell M, Mora N, Vlcek F, Kutaj-Wasikowska H, Kutryba B, Michel P, Groene O. 2014. Organización basada en la evidencia y estrategias de seguridad del paciente en los hospitales europeos. Int. J. Qual Health Care. Supl. 1.Recuperado de: http://www.ncbi.nlm.nih.gov/ pubmed/24578501 